\title{
Atypical presentations of tuberculous meningitis patient on prolong mechanical ventilation: - A CASE REPORT
}

\author{
Dr. Anil k Paswan ${ }^{1}$, Dr. RK.Dubey ${ }^{1}$ 'Sandeep Khuba ${ }^{1}$, Suman sarkar ${ }^{2}$ \\ MBBS, MD, Assistant prof. Department of anaesthesial, senior Resident2, IMS, BHU, Varanasi. India
}

\begin{abstract}
Tracheal stenosis is the most common late airway complication of post intubation/trachestomy resulted in hemodynamic deterioration and impairment of respiratory system mechanics. Post-dural puncture headache (PDPH) is well known complication of introduction of spinal/epidural needle in subarachanoid space. In this article, we present a very rare case report of a patient 18 year old male, diagnosis tuberculous meningitis(TBM) get prolong mechanical ventilation and dural puncture had developed tracheal stenosis and post-dural puncture headache . We describe a patient with PDPH and tracheal stenosis which was initially misdiagnosed as simple headache and asthma after prolonged tracheal intubation. Surgery is lifesaving for patients with critical tracheal stenosis, but how to ensure effective gas exchange is crucial to the anesthetic management.
\end{abstract}

KEY WORD:-TBM .PDPH .TRACHEAL STENOSIS

CASE HISTORY:-

A 18 year-old male who presented with a 6-week history of headache and fever and a 8-hour history of inability to speak and alteration in level of consciousness. There was history of neck pain, occasional nausea /vomiting and no neck stiffness .He had no other remarkable past history of medical and surgical illness. Before presentation, he had been kept on various intravenous antibiotics in private hospitals, with no clinical improvement. Physical examination showed a young man in an altered level of consciousness with signs of meningeal irritation and had left spastic hemiparesis. The patient had progressive respiratory distress with rapid shallow breathing, while using all accessory muscles of respiration. He had a respiratory rate of 35 breaths $\mathrm{min}^{-1}$, and a fluctuating arterial oxygen saturation of 70 80\% with $10 \mathrm{~L} \cdot \mathrm{min}^{-1}$ of supplementary oxygen. The patient was ventilated with the synchronized intermittent mandatory ventilation with volume controlled mode selected. The ventilatory settings were as follows: $\mathrm{FiO}_{2} 0.40$; respiratory rate 14 breaths $/ \mathrm{min}$; Tidal volume $500 \mathrm{ml}$; pressure support $12 \mathrm{~cm}$ $\mathrm{H}_{2} \mathrm{O}$; positive end expiratory pressure (PEEP) $5 \mathrm{~cm} \mathrm{H}_{2} \mathrm{O}$. With above mentioned settings, the peak airway pressure (PIP) was $16 \mathrm{~cm} \mathrm{H}_{2} \mathrm{O}$, and the arterial blood gas parameters were satisfactory.A lumbar puncture was performed with $23 \mathrm{G}$ spinal needle and produced opalescent cerebrospinal fluid (CSF) with CSF pressure of $14 \mathrm{~cm}$ of $\mathrm{H} 20$. The biochemical/microbiological features of CSF are :- Protein $80 \mathrm{mg} / \mathrm{dl}$; glucose $40 \mathrm{mg} / \mathrm{dl}$; WBC $75 / \mathrm{micro} \mathrm{L}$, chiefly there was Lymphocyte (70\%)and CSF acid fast and grams staining were negative . CSF culture was not sent .The computed tomography scan of brain showed obstructive hydrocephalus. These above mentioned feature was suggestive of tuberculous meningitis .He was placed immediate on antituberculous drugs and intravenous steroids and mannitol .He was successfully weaned off mechanical ventilatory support after 10 days. After first day of weaning patient experienced of headache and vertigo. Intensivist assumed that headache and vertigo is a part of TBM and symptoms will subside after few days course of ATT but symptoms get more severed and could not get relieved even after analgesic, antivertigo and ATT medication. The neurological examination revealed that the upper and lower extremity strength was 5 of 5 bilaterally. There were no meningeal signs or papilledema. Then patient referred to neurology and ENT department but could not get relieved even after 20 days of illness. lastly intensivist took a consultation from anaesthesiology department and found that there was typical history of PDPH, complaints of pain was dull, nonthrobbing pain in fronto-occipital region, which was aggravated in the sitting and standing position and reduce in the supine position and make a planned for autologous epidural blood patch, given $20 \mathrm{ml}$ blood and kept on bed ridden for $12 \mathrm{hrs}$ and get improved. After 8- weeks of ATT, again complained of exertional dyspnoea and wheeze receiving nebulised bronchodilators, intravenous hydrocortisone, aminophylline, and antibiotics. In spite of above mentioned treatment patient continued to deteriorate and became exhausted and referred to intensive care unit for further management had tachypnoea (respiratory rate 30/min), hypercarbia and hypoxemia $\left(\mathrm{Paco}_{2-}\right.$ $80 \mathrm{mmhg}$ and $\mathrm{PaO}_{2}$ - 55mmhg) on arterial blood gas analysis. We decided to intubate and ventilate him. After intravenous induction of anaesthesia, laryngoscopy was performed, but it was impossible to pass adult size endotracheal tube further than $1.5 \mathrm{~cm}$ beyond his vocal cords in spite of a good view of a normal glottis. Even does not pass s a flexible gum elastic boogie beyond the obstruction. We managed to ventilate him adequately with a bag, valve, and mask. We decided to perform to fiberoptic bronchoscopy and find out there was severe tracheal stenosis (2- 3mm) (fig.1) and it does not pass beyond $1.5 \mathrm{~cm}$ 
of vocal cord and regularly we managed to ventilate with bag and mask with slight improvement in Spo2 92\%. What was severity and length of tracheal stenosis, we don't know, so unable to performed tracheostomy and continued to ventilate with bag an mask. A computed tomography scan of neck revealed that the Heterogeneous mucosal thickening with patchy area of calcification, involving upper part of trachea approx. $2.5 \mathrm{~cm}$ length extending below cricoid cartilage, suggestive of upper Tracheal stenosis( fig.2 and 3). And confirmed that have severe post intubation tracheal stenosis secondary to prolonged mechanical ventilation. Emergency tracheostomy done quickly after fiberoptic bronchoscopy and CT scan of neck. Finally ENT surgeon placed a stent endoscopically after resection and dilation of tracheal mucosa.

DISCUSSION-: In August 1898, Karl August Bier, ${ }^{1}$ a German surgeon, and his assistant injected cocaine 10-15 mg into the subarachnoid space of seven patients .four out of seven patient, complaint headache and thus Bier, and his assistant described the symptoms post-dural puncture headache due to loss of CSF. The rate of CSF loss through the dural perforation $\left(0.084-4.5 \mathrm{ml} \mathrm{s}^{-1}\right)$ is generally greater than the rate of CSF production $\left(0.35 \mathrm{ml} \mathrm{min}{ }^{-1}\right)$, specially with needle sizes larger than $25 \mathrm{G}^{2}$ The incidence of PDPH a $<2 \%$ with a $29 \mathrm{G}$ needle; $2 \%-12 \%$ with a $26 \mathrm{G}$ Quincke needle; $25 \%$ with a $25 \mathrm{G}$ needle; ${ }^{3,4}$ $40 \%$ with a $22 \mathrm{G}$ needle and PDPH is usually a self-limiting process, but Vandam and Dripps in 1956 reported that $72 \%$ of headaches resolved within a week ${ }^{5}$. PDPH usually responds to increased fluid intake, bed rest and analgesics. Among therapeutic strategies, epidural blood patch is most effective and it has been recommended for treatment of persistent headache after dural puncture. Epidural blood patch prevent headache by different mechanism 1) presence of clot at dural hole to prevent CSF leak 2) Compression of the epidural space and elevation of subarachnoid pressure 3) CSF acts as procoagulant and accelerate clotting process. Epidural Hartmann's solution, epidural dextran and saline has been reported to reduce $\mathrm{PDPH}^{6}$.it has been suggested that placement of a spinal catheter( through the accidental dural perforation with a Tuohy needle), may provoke an inflammatory reaction which that will seal the hole. ${ }^{7}$ Caffeine, sumatriptapan and theophylline has been shown to beneficial in relieving PDPH by cerebral vasoconstriction effect ${ }^{8-10}$. Another non invasive method to treat PDPH are bed rest, hydration ,abdominal binder, analgesics, and oral Gabapentine $400 \mathrm{mg}{ }^{11}$ tid has been reported to relieves pain within $24 \mathrm{hrs}$.. It is possible that postdural puncture headache left untreated may be complicated by the development of subdural hematoma. ${ }^{12}$ Patients developing a postdural puncture headache unrelieved by conservative measures, then we should plan for invasive measure.

Tracheal stenosis is rare and late serious complication of prolong ventilation and misdiagnosed as bronchial asthma. Tracheal injury, either iatrogenic or traumatic, mostly heals with some degree of tracheal stenosis. Incidence of prolonged intubation ranges from 10 to $22 \%$ but only $2-3 \%$ of the patients are symptomatic have severe stenosis ${ }^{13}$. Generally, when the patient presents stridor and tachypnea, the tracheal stenosis has reached about $50 \%$ of the tracheal diameter. When the patient has extreme respiratory insufficiency the tracheal stenosis has reached a critical level of $75 \%$ or more. After endotracheal intubation most common sites of tracheal stenosis occur where the endotracheal tube cuff is in contact with the tracheal wall and at the level of tracheal stoma site after a tracheostomy. The stenosed position is also important for anaesthesia, besides the degree of tracheal stenosis. For upper tracheal stenosis, a small tracheal tube can be inserted below the stenosis with the help of bronchoscopy. Incidence of Tracheal stenosis can be minimised by the use of high volume and low pressure cuff pressure should be less than $25 \mathrm{mmhg} \mathrm{Hg}$. If endotracheal tube cuff pressure more than $25 \mathrm{~mm} \mathrm{Hg}$ exceeds the mucosal capillary perfusion pressure, which may leads to mucosal ischemia, ulceration granulation tissue and scar formation which may lastly develop tracheal stenosis ${ }^{13}$. This is now standard practice the use high volume, low pressure cuffs is on intensive care units. In spite of that tracheal stenosis may occur and in one prospective study of critically ill patients, $11 \%$ of patients who had been intubated with high volume, low pressure cuffed tubes developed tracheal stenosis that were 10$50 \%$ of their tracheal diameter at the cuff site. ${ }^{14}$.

CONCLUSION:-Most common cause of PDPH is use of larger spinal needle .so must be use smaller size needle to prevent PDPH. Mild to moderate PDPH best treated by conservatively as the natural history of disease resolved within a week. Acquired subglottic/tracheal stenosis is typically more severe urgently require surgical intervention. It is difficult to treat and open surgical method is associated with significant risk and complications. Routinely endoscopic control of trachea should be carried out in intensive care unit especially in prolong intubated patient symptomatic as well as asymptomatic patient.

\section{REFERENCES.}

1. Wulf HF. The centennial of spinal anesthesia. Anesthesiology 1998; 89: 500-6

2. Cruickshank RH, Hopkinson JM. Fluid flow through dural puncture sites. An in vitro comparison of needle point types. Anaesthesia 1989; 44: 415-18 
3. Barker P. Headache after dural puncture. Anaesthesia 1989; 44: 696-714

4. Flaatten H, Rodt S, Rosland J, Vamnes J. Postoperative headache in young patients after spinal anaesthesia. Anaesthesia 1987; 42: 202-5

5. Vandam LD, Dripps RD. Long-term follow up of patients who received 10098 spinal anesthetics. JAMA 1956; 161: 586-91

6. Crawford JS. The prevention of headache consequent upon dural puncture. Br J Anaesth 1972; 44: 598-600

7. Denny N, Masters R, Pearson D, Read J, Sihota M, Selander D. Postdural puncture headache after continuous spinal anesthesia. Anesth Analg 1987; 66: 791-4

8. Schzer PH, Abel H. Post spinal anesthesia headache treated with caffeine, Evaluation with demand method. Part I.Curr TherpRes 1978; 24: 307-12.).9 Schwalbe SS, Schifmiller MW, Marx GF. Theophylline for PDPH Anesthesiology 1991; 75: A1082.)

10. Carp H, Singh PJ et al. Effects of serotonin-receptor agonist sumatriptan on PDPH: Report of six cases Anesth Analg 1994;79: 180-84.)

11. Lin YT, Sheen MJ, Huang ST, et al .Gabapentin relieves post-dural puncture headache- a report of two cases. Acta Anaesthesiol Taiwan. 2007 Mar;45:47-51

12. A. Zeidan, O. Farhat, H. Maaliki and A. Baraka Does postdural puncture headache left untreated lead to subdural hematoma? Case report and review of the literature international journal of obstetric anesthesia 2006; 15:50-58

13. Sue RD, Susanto I. Long-term complications of artificial airways. Clin Chest Med 2003;24(3):457-471)

14. Stauffer JL, Olson DE, Petty TL. Complications and consequences of endotracheal intubation and tracheotomy: a prospective study of 150 critically ill adult patients. Am J Med 1981;70(1):65-76).

Figure1. severe subglottic stenosis shown on fiberoptic bronchoscopy 


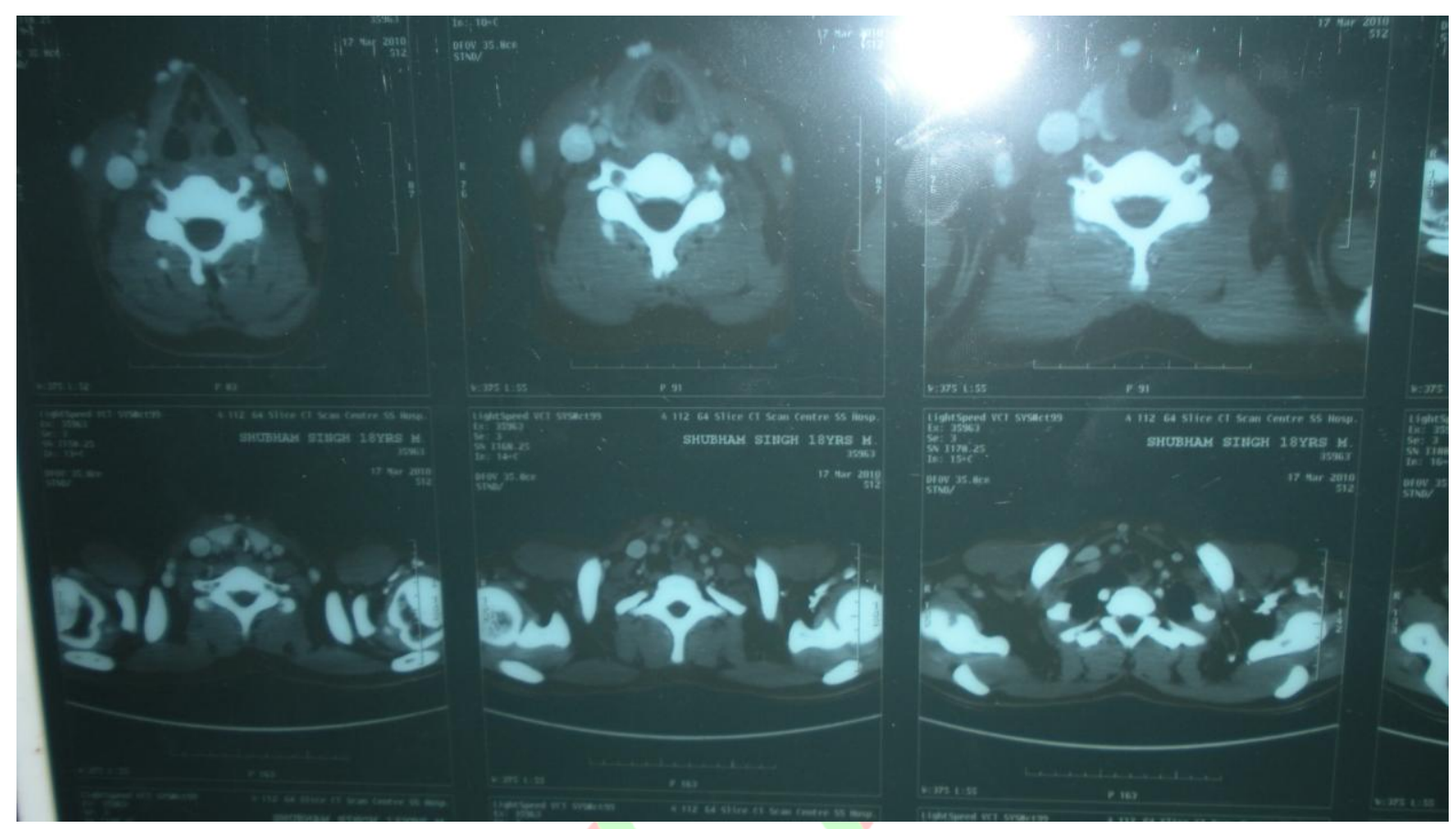

Figure 2.Tracheal stenosis shown on CT scan

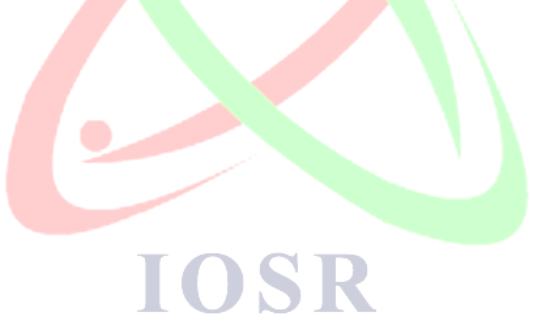




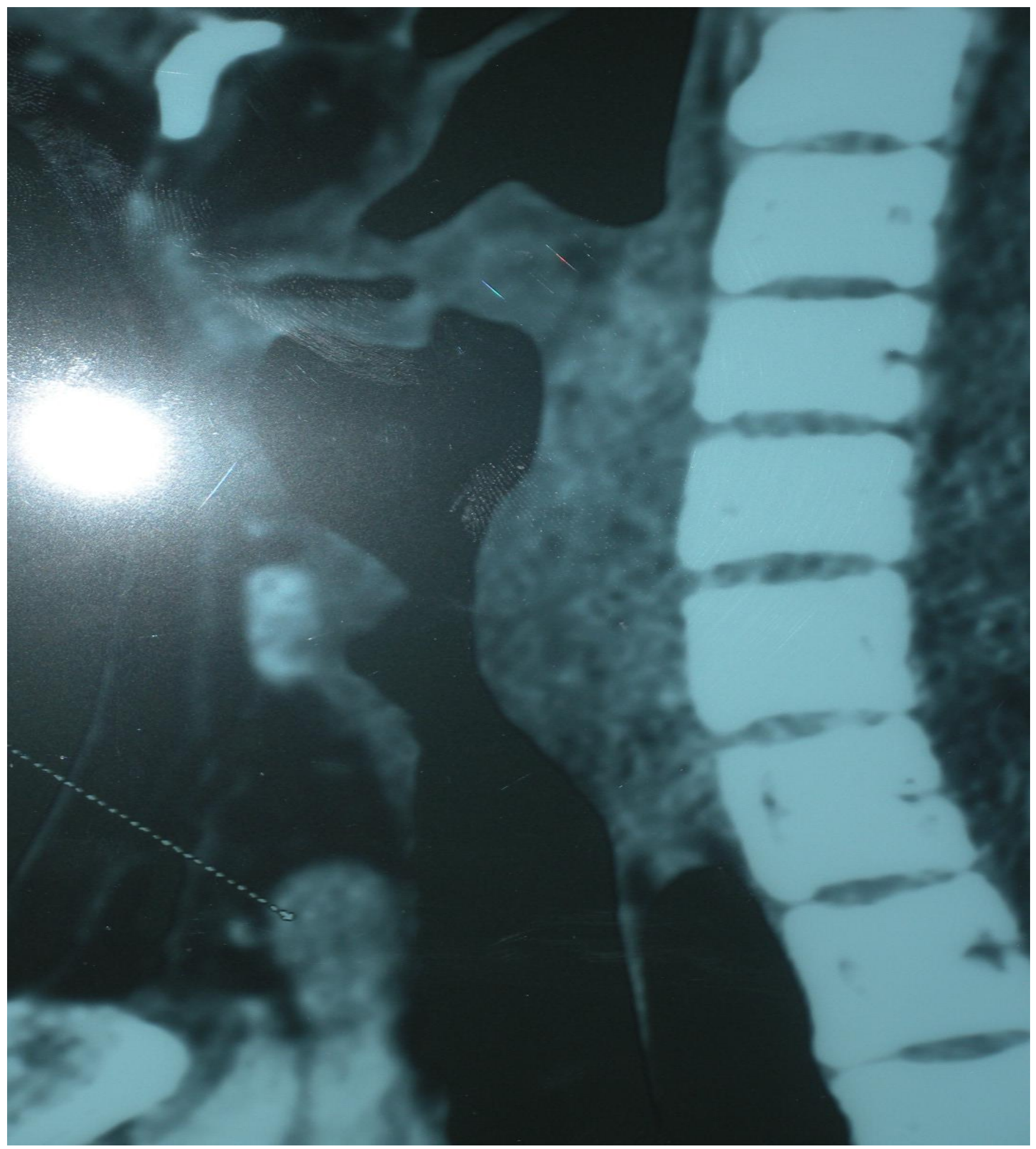

Figure 3.CT Scan shown heterogeneous mucosal thickening with patchy area of calcification, involving upper part of trachea approx. $2.5 \mathrm{~cm}$ length extending below cricoid cartilage? Upper Tracheal stenosis 